\section{JTI}

JOURNAL OF

TRAUMA AND INJURY

\title{
Clinical Analysis of the Patients with Isolated Low-Velocity Penetrating Neck Injury
}

\author{
Junepill Seok, M.D., Hyun Min Cho, M.D. \\ Department of Trauma and Critical Care Surgery, Pusan National University Hospital, Busan, Korea
}

Received: November 8, 2017

Revised: November 23, 2017

Accepted: December 4, 2017

\section{Correspondence to}

Hyun Min Cho, M.D.

Department of Trauma and Critical Care Surgery, Pusan National University Hospi-

tal, 179 Gudeok-ro, Seo-gu, Busan 49241, Korea

Tel: $+82-51-240-7369$

Fax: +82-51-240-7719

E-mail:suc2601@gmail.com

*This work was supported by clinical research grant from Pusan Naional University Hospital in 2017.

Purpose: Although there has been substantial progress for the treatment of thoracic trauma, the mortality of the penetrating neck injury is still high, has been reported about $10-15 \%$. However, there has not been a report which is reflecting Korean medical present. We retrospectively analyzed the penetrating neck injury patients based on the Korean Trauma Data Base.

Methods: Between December 2013 and June 2017 at the trauma center of the Pusan National University Hospital, Busan, Korea, total of 36 patients with isolated low-velocity penetrating neck injuries were included. We analyzed the patients' age, gender, injury mechanism and causes by medical chart review.

Results: Among total of 36 patients, 26 (72.2\%) were male and 10 (27.8\%) were female. Homicidal neck injuries were most common, followed by accidental and suicidal injuries ( $47 \%$ vs. $33 \%$ vs. $19 \%$, respectively). All penetrating injuries in our study were low-velocity trauma such as following: knife $(\mathrm{n}=16,44.4 \%)$; glass or glass bottle $(\mathrm{n}=11$, $30.6 \%)$; scissors $(n=4,11.1 \%)$; grinder $(n=2,5.6 \%)$; and three $(8.3 \%)$ of miscellaneous injuries. Twenty-seven (75.0\%) patients underwent emergency surgery, and only one (2.8\%) patient underwent elective surgery. Eleven (30.6) patients were diagnosed with superficial injuries, including six patients who had conservative treatment. Twelve (33.3\%) patients had arterial injuries and $10(27.8 \%)$ patients had venous injuries. The patients who had deep injuries showed significant difference against the patient with superficial injury (98.0 vs. 129.1, $p=0.008$ ).

Conclusions: Low velocity penetrating injury confined to the neck is able to be successfully treated with prompt surgical management. Regardless of the conditions which are evaluated at emergency department, all penetrating neck injury patients should be regarded as urgent surgical candidates.

Keywords: Trauma; Penetrating; Stab; Neck

This is an Open Access article distributed under the terms of the Creative Commons Attribution Non-Commercial License (http://creativecommons.org/licenses/by-nc/4.0/) which permits unrestricted noncommercial use, distribution, and reproduction in any medium, provided the original work is properly cited. 


\section{INTRODUCTION}

Penetrating neck injuries are a great challenge for medical professionals. The most common mechanism of injury worldwide is a stab wound from violent assault, followed by gunshot wounds, suicide, road traffic accidents and other high velocity objects [1,2].

Although there has been substantial progress for the treatment of thoracic trauma, the mortalities are still high, because important organs such as trachea, esophagus, great vessels, and nerves are crowded in small space. In the USA, the mortality of the penetrating neck injury has been reported about 10-15\% [3], however, this result is not applicable to Korea because gunshot injuriese extremely rare.

In this report, to identify characteristics of the penetrating neck injuries, we retrospectively analyzed the patients registered in the Korean Trauma Data Base who were admitted to the trauma center of Pusan National University Hospital from 2013 to 2017.

\section{METHODS}

We retrospectively reviewed the trauma registry and medical records between December 2013 and June 2017 at the trauma center of the Pusan National University Hospital, Busan, Korea. Our trauma center is categorized as a Level 1 trauma center by the Ministry of Health and Welfare. We tried to include the patients with isolated neck injury. Among total of 7,670 trauma patients who admitted to our hospital in this period, 36 patients were finally sorted. We defined the patients who only had injuries of the skin and muscles as superficial, and the patients with vascular or deep structural injuries such as bone, cartilage and cervical nerves as deep injury. Computed tomography (CT) was performed if the patients had relatively acceptable systolic blood pressure (SBP) after initial resuscitation. Despite of vigorous fluid infusion, if patients were still hemodynamic unstable, prompt surgical exploration was performed. Penetrating neck injury can damage to the vessels as well as deep structures, we preferred to perform exploration rather than radiologic intervention except a very special case.
Statistical analyses were performed using SPSS 22.0 (IBM Corp., Armonk, NY, USA). Continuous data were presented as median (interquartile range); categorical data were presented as $\mathrm{n}(\%)$. A $\chi^{2}$, Fisher's exact test and analysis of variance (ANOVA) by using Turkey's method were used to assess associations between categorical variables.

\section{RESULTS}

Baseline characteristics are described in Table 1. Among total of 36 patients, $26(72.2 \%)$ were male and 10 (27.8\%) were female. Mean age was 54.3 (standard deviation [SD] 17.3). Homicidal neck injuries were most common, followed by accidental and suicidal injuries (47\% vs. $33 \%$ vs. $19 \%$, respectively). Mean injury severity score (ISS) of 35 patients was 8.3 (SD 8.1), except 1 patient who died at emergency department (ED) before being evaluated. All penetrating injuries in our study were low-velocity trauma such as following: knife $(\mathrm{n}=16,44.4 \%)$; glass or glass

Table 1. Baseline characteristics

\begin{tabular}{|lc|}
\hline & Value $(\mathbf{n}=\mathbf{3 6})$ \\
\hline Gender & $n=36$ \\
Male & $26(72.2)$ \\
Agemale & $10(27.8)$ \\
Causes of trauma & $54.3(17.3)$ \\
Homicidal & \\
Accident & $17(47)$ \\
Suicidal & $12(33)$ \\
Mortality & $7(19)$ \\
Injury Severity Score & $1(2.8)$ \\
Applicable $(n=35)$ & \\
Unknown $(n=1)$ & $8.3(8.1)$ \\
Injury mechanism & \\
Knife & \\
Glass, glass bottle & $16(44.4)$ \\
Scissors & $11(30.6)$ \\
Grinder & $4(11.1)$ \\
Miscellaneous & $2(5.6)$ \\
Values & $3(8.3)$ \\
\hline
\end{tabular}

Values are presented as number (\%). N/A: not applicable. 
Table 2. The degree of injury and treatment results

\begin{tabular}{|lc|}
\hline & Value \\
\hline Emergency operation & $2775.0)$ \\
Elective operation & $1(2.8)$ \\
Angiography & $1(2.8)$ \\
Expired & $6(16.7)$ \\
Superficial injury & $1(2.8)$ \\
Deep injury & $11(30.6)$ \\
Arterial & $25(69.4)$ \\
Facial artery & $12(33.3)$ \\
Thyroid artery & $4(11.1)$ \\
Common carotid artery & $2(5.6)$ \\
External carotid artery & $3(8.3)$ \\
Internal carotid artery & $3(8.3)$ \\
Venous & $0(0.0)$ \\
External jugular vein & $10(27.8)$ \\
Internal jugular vein & $8(22.2)$ \\
Thyroidal vein & $4(11.1)$ \\
Miscellaneous & $1(2.8)$ \\
Cervical nerves & \\
Thyroid cartilage & $3(8.3)$ \\
\hline
\end{tabular}

Values are presented as number (\%).

bottle $(\mathrm{n}=11,30.6 \%)$; scissors $(\mathrm{n}=4,11.1 \%)$; grinder $(\mathrm{n}=2$, $5.6 \%)$; and $3(8.3 \%)$ of miscellaneous injuries.

Table 2 describes the degree of injury and the results of treatment. Among total of 36 patients, $27(75.0 \%)$ patients underwent emergency surgery, and only one (2.8\%) patient underwent elective surgery. Six (16.7\%) patients only had superficial laceration and were sutured at ED. A patient who had rupture of the vertebral artery due to acupuncture therapy at a local clinic was successfully treated with angiographic embolization. Eleven (30.6\%) patients were diagnosed with superficial injuries, including six patients who had conservative treatment. Five (13.9\%) superficial neck injury patients who underwent surgery only had muscular bleedings without deeper structural injuries. Twelve (33.3\%) patients had arterial injuries such as following: facial artery $(\mathrm{n}=4,11.1 \%)$; thyroid artery $(\mathrm{n}=2,5.6 \%)$; common carotid artery $(\mathrm{n}=3,8.3 \%)$; and external carotid artery $(n=3,8.3 \%)$. No patient had injured internal carotid artery. Ten $(27.8 \%)$ patients had venous
Table 3. Differences of systolic blood pressure between variables

\begin{tabular}{|lccc|}
\hline & No. & SBP, mean (SD) & $\boldsymbol{p}$ \\
\hline Superficial injury & 11 & $129.1(24.3)$ & \\
Deep injury & 25 & $98.0(41.3)$ & 0.008 \\
$\quad$ Arterial bleeding & 12 & $93.6(47.9)$ & 0.037 \\
Venous bleeding & 10 & $93.0(32.7)$ & 0.009 \\
Miscellaneous & 4 & $114.3(31.0)$ & 0.346 \\
\hline
\end{tabular}

No.: number, SBP: systolic blood pressure, SD: standard deviation.

Table 4. Analysis of variance (ANOVA) for injury mechanisms

\begin{tabular}{|lccccc|}
\hline Variables & $\mathbf{n}$ & SBP & $\mathbf{9 5 \% ~ C l}$ & $p^{\text {a }}$ & $p^{\mathbf{b}}$ \\
\hline Homicidal & 17 & $90.8(39.7)$ & $70.4-111.3$ & 0.011 & 0.627 \\
Accidental & 12 & $132.2(26.1)$ & $115.7-148.9$ & & \\
Suicidal & 7 & $105.7(39.5)$ & $69.1-142.2$ & & \\
\hline
\end{tabular}

SBP: systolic blood pressure; Cl: confidence interval.

asignificance between homicidal and accidental.

${ }^{\text {b}}$ Significance between homicidal and suicidal.

injuries such as following: external jugular vein $(n=8$, $22.2 \%)$; internal jugular vein $(\mathrm{n}=4,11.1 \%)$; and thyroidal vein $(n=1,2.8 \%)$. Four (11.1\%) patients had deep injuries besides of vascular damages, among them two (5.6\%) patients had cervical nerve dissection without vessel injury.

Table 3 describes the differences of SBP between the subgroups of patients. SBP of the patients with deep injuries were significantly lower when compared to that of patients with superficial neck injury (98.0 vs. 129.1, $p=0.008$, respectively). Specifically, SBPs in the cases of the arterial and venous injuries were significantly lower than that of the superficial injury (93.6 vs. 129.1, $p=0.037$; 93.0 vs. $129.1, p=0.009$, respectively). Aforementioned, four (11.1\%) patients had deep structural injuries such as following: three $(8.3 \%)$ of the cervical nerve injury; one $(2.8 \%)$ of the thyroid cartilage injury, among them two (5.6\%) patients with cervical nerve injury did not accompany vascular damage. In this group, SBP did not show statistical significance compared to that of the patients with superficial injuries (114.3 vs. 129.1, $p=0.346$, respectively).

Table 4 describes ANOVA test for the differences of SBP among three causes of neck injury; homicidal; accidental and suicidal. In this analysis, SBP of the patients 
who had homicidal attack was significantly lower than that of the patients who had accidental neck injury ( 90.8 vs. $132.2, p=0.011$, respectively), in other hand, there were no differences of SBP against the suicidal group.

\section{DISCUSSION}

The mechanism of penetrating injury may be categorized as low, medium, or high velocity. Low-velocity injuries include impalement such as knife wounds, which disrupts only the structures penetrated. Medium-velocity injuries include bullet wounds from most types of handguns and air-powered pellet guns and are characterized by much less primary tissue destruction than wounds caused by high-velocity forces. High-velocity injuries include bullet wounds caused by rifles and wounds resulting from military weapons [4]. In the case of Korea, most of penetrating injuries are low velocity mechanisms by stabbing and medium or high velocity injuries by GSW are extremely rare. In this report, we tried to identify characteristics of the penetrating neck injuries which are reflecting the present of Korean medical environment. As a result, there was no high or medium velocity trauma (Table 1) among 36 patients with isolated neck penetrating injuries. According to previous study [5], low-velocity penetrating injury has lower mortality than the others. In our study, only one patient died at ED among total of 36 patients who admitted to our trauma center, even the patient did not have enough time to go operating room.

Various algorithms to treat penetrating neck trauma have been introduced. Due to differences between the cultures, medical instruments setting of hospitals, and the incidences, not all algorithms are same, but mostly are similar $[2,6,7]$. They advocated that nontherapeutic neck exploration rate can be reduced by following proper protocols, however, we have not experienced with enough cases to make prompt decisions. As a result, we preferred to perform explorations to the patients. In our study, among the total of 36 patients, 27 (75.0\%) patients underwent surgical exploration and five patients were diagnosed with superficial injury without major organ damage. Literally our nontherapeutic neck exploration rate was $18.5 \%$ ( 5 cases of negative findings among 27 explorations), however, it was relatively acceptable result when compared to previous studies [2,7]. Additionally, Low et al. [8] demonstrated in 2014 a poor correlation between the location of the external wound and the injuries to internal structures. Also, with development of Multi-dimensional CT angiography, the non-zonal approach is superior over traditional anatomical zones management algorithms [7]. The initial SBP, features of the wound such as active bleeding, and the depth of injuries which was initially measured at ED were the major variables to decide whether perform a surgical exploration or not in our study. We did not regard the anatomical zones meaningfully.

As shown at Table 3 and Table 4, aggressive and prompt surgical explorations are required for penetrating neck injury especially if a patient's SBP was lower or if a patient is attacked or attempted suicide. However, SBP is not only the diagnostic criteria. Aforementioned, 2 hemodynamic stable patients had cervical nerve injuries without vascular damage. With considering that it is difficult to perform precise physical examinations at ED, aggressive neck exploration despite of hemodynamic stability can decrease the morbidity and mortality if surgeons are not fully experienced with penetrating neck injuries. In our hospital, among 7,670 trauma patients who admitted in study period, lesser than $5 \%$ patients had penetrating trauma and $0.5 \%$ patients had isolated penetrating neck trauma. We are trying to establish surgical algorithm which is well reflecting our medical present.

We performed one case of endovascular stenting to treat vertebral arterial pseudoaneurysm. In this case, the vertebral artery had been damaged by an acupuncturist before visiting our hospital. Therefore we easily excluded the possibility of deep structural damage and decided to perform radiologic intervention. Many reports have mentioned that radiologic intervention can be performed safely and effectively for selective cases of penetrating neck injury $[9,10]$, however, it is dependent to the instrumental setting of hospitals and the reports for long-term outcome have been little. Authors guess that the role of the radiologic intervention for the penetrating neck injury is confined, and applicable to only selective cases. 


\section{CONCLUSION}

Although penetrating neck injuries are a great challenge for medical professionals, however, low velocity penetrating injury confined to the neck is able to be successfully treated with prompt surgical management. Regardless of the conditions which are evaluated at ED, all penetrating neck injury patients should be regarded as urgent surgical candidates.

\section{REFERENCES}

1. Burgess CA, Dale OT, Almeyda R, Corbridge RJ. An evidence based review of the assessment and management of penetrating neck trauma. Clin Otolaryngol 2012;37:44-52.

2. Mahmoodie M, Sanei B, Moazeni-Bistgani M, Namgar M. Penetrating neck trauma: review of 192 cases. Arch Trauma Res 2012;1:14-8.

3. Thompson EC, Porter JM, Fernandez LG. Penetrating neck trauma: an overview of management. J Oral Maxillofac Surg 2002;60:918-23.

4. Kuhajda I, Zarogoulidis K, Kougioumtzi I, Huang H,
Li Q, Dryllis G, et al. Penetrating trauma. J Thorac Dis 2014;6(Suppl 4):S461-5.

5. Khandhar SJ, Johnson SB, Calhoon JH. Overview of thoracic trauma in the United States. Thorac Surg Clin 2007;17:1-9.

6. Sperry JL, Moore EE, Coimbra R, Croce M, Davis JW, Karmy-Jones R, et al. Western trauma association critical decisions in trauma: penetrating neck trauma. J Trauma Acute Care Surg 2013;75:936-40.

7. Nowicki JL, Stew B, Ooi E. Penetrating neck injuries: a guide to evaluation and management. Ann R Coll Surg Engl 2017:1-6.

8. Low GM, Inaba K, Chouliaras K, Branco B, Lam L, Benjamin E, et al. The use of the anatomic 'zones' of the neck in the assessment of penetrating neck injury. Am Surg 2014;80:970-4

9. Du Toit D, Coolen D, Lambrechts A, Odendaal JdV, Warren BL. The endovascular management of penetrating carotid artery injuries: long-term follow-up. Eur J Vasc Endovasc Surg 2009;38:267-72.

10. Kim JP, Park JJ, Won SJ, Woo SH. Penetrating carotid artery injuries treated by an urgent endovascular stent technique: report of two cases. Chonnam Med J 2011;47:134-7. 